\title{
Composition, structure and function of the eukaryotic flagellum distal tip
}

\author{
Jacob T. Croft ${ }^{1, *}$, Davide Zabeo ${ }^{1, *}$, Radhika Subramanian² and (1D Johanna L. Höög1 \\ ${ }^{1}$ Department of Chemistry and Molecular Biology, University of Gothenburg, Gothenburg, Sweden; ${ }^{2}$ Department of Molecular Biology, MGH and Department of Genetics, Harvard \\ Medical School, Boston, MA 02114, U.S.A.
}

Correspondence: Johanna L. Höög (johanna.hoog@gu.se)

\section{OPEN ACCESS}

Cilia and flagella are long extensions commonly found on the surface of eukaryotic cells. In fact, most human cells have a flagellum, and failure to correctly form cilia leads to a spectrum of diseases gathered under the name 'ciliopathies'. The cilium distal tip is where it grows and signals. Yet, out of the flagellar regions, the distal tip is probably the least intensively studied. In this review, we will summarise the current knowledge on the diverse flagellar tip structures, the dynamicity and signalling that occurs here and the proteins localising to this important cellular region.

\footnotetext{
* These authors contributed equally to this manuscript.
}

Received: 04 July 2018 Revised: 02 October 2018 Accepted: 05 October 2018 21 November 2018

\section{Introduction}

Protruding into the extracellular environment, the cilium (aka flagellum) is a microtubule-based organelle located at the cell surface [1]. Many unicellular protists as well as cells in complex multicellular organisms such as humans have a flagellum in this exposed position (Figure 1), where it is a crucial tool for cellular signalling and locomotion. The distal tip of the flagellum is the epicentre of much of its signalling and its assembly [2-7], yet we know very little of its composition and structure, especially in humans. If cilia do not form properly, the consequences are dire - a whole group of diseases called the ciliopathies [8]. What is this mysterious organelle that is so important to cellular function?

Inside the flagellar membrane there is a specialised structure composed of nine microtubule doublets arranged in a circle (Figure 2A). Each doublet microtubule consists of a complete 13 protofilament A-tubule and an incomplete 10 protofilament B-tubule [9]. Inside motile flagella there are two additional singlet microtubules in the middle, the central pair, that are thought to be involved in the regulation of the flagellar beat [10]. The beat occurs as thousands of dynein motor proteins walk on one microtubule while being attached to the neighbouring microtubule, causing it to bend. The microtubules together with the associated structural proteins are called the axoneme. The complexity of this machine is shown in proteomics studies that find that it is composed of approximately 400-1000 different proteins [11-13]. Sensory cilia usually lack dynein motors, the central pair microtubules and their associated proteins and have therefore a smaller proteome of approximately $200-400$ proteins $[14,15]$. What fraction of the cilium proteome resides at the distal tip is currently unknown.

The flagellum can be further divided into multiple zones along its length (Figure 2B). Beginning in the cytoplasm, there is the proximal end of the flagellum, the basal body. The basal body has nine triplet microtubules, of which only the A- and B-tubules extend into and beyond the transition zone where usually a basal plate or other electron-dense structures are found. It is also in the transition zone that the central pair microtubules start in motile flagella. The axoneme then extends for approximately $2.5-50 \mu \mathrm{m}$, depending on the species and cell type (for a review on the whole flagella structure, see [16]). In this review, we will focus on the distal most tip of the flagellum, a specialised region full of cellular activity. 

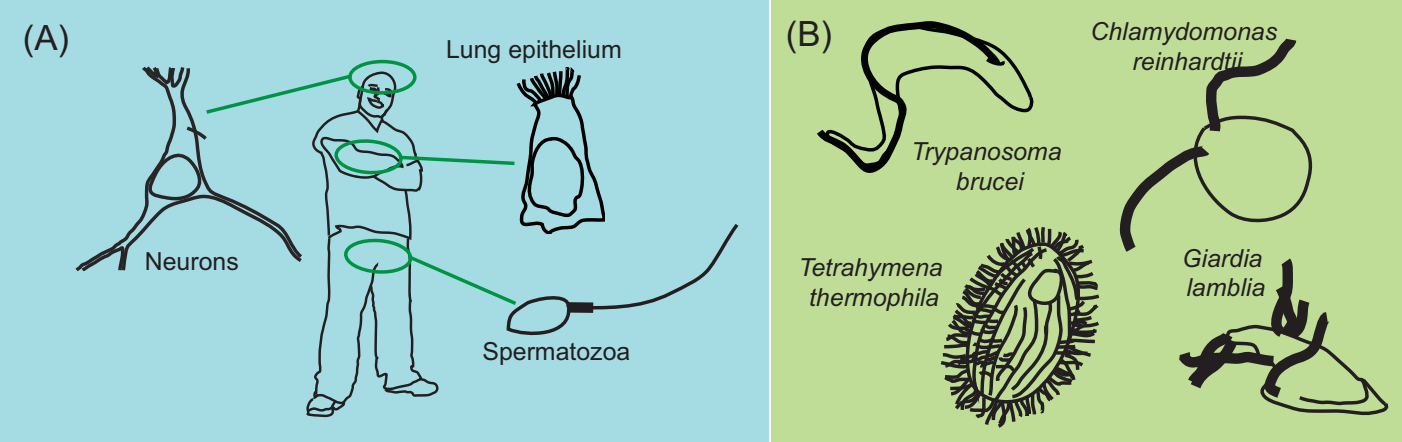

Figure 1. Examples of ciliated cells in both multicellular organisms such as humans (A) and unicellular organisms (B).

\section{Structural models for distal tips of motile flagella}

Most studies on flagellar tips focused on protists like the green alga Chlamydomonas reinhardtii, the ciliate Tetrahymena thermophila and the human parasite Trypanosoma brucei. However, the ultrastructure of flagellar tips has been investigated in many different organisms, revealing considerable differences. We sorted the reported flagella tip structures into six different models (Figure 3).

Model 1: Electron microscopy (EM) of C. reinhardtii flagellar tips on demembranated negatively stained samples showed a large capping structure, formed by two plate-like structures and an electron-dense bead at the distal ends of central pair microtubules. It was termed as central microtubule cap [17,22]. Smaller carrot-like plugs capped the A-tubules in doublet microtubules, by extending into their lumen. Distal filaments connected the plugs and the central microtubule cap to the plasma membrane of the flagellar tip in multiple spots.

Model 2: Thin sections of chemically fixed C. reinhardtii flagella revealed an approximately 400-nm long singlet region, which was characterised by the absence of radial spokes and by the gradual termination of the doublets (B-tubules terminated before the A-tubules) [23]. The bead of the central microtubule cap was not observed in images of sectioned flagellar tips and only the two plates were left in association with the central pair microtubules [18,23-26]. Instead, an electron density was observed to laterally connect the distal segments of the two central pair microtubules. Flagella of bay scallop gills also have this tip arrangement, but here distal filaments were seen to connect A-tubules to the central microtubule cap [22]. Only undefined densities were observed instead of a structured central pair cap in electron tomography (ET) of high-pressure frozen and freeze substituted (HPF-FS) growing C. reinhardtii flagellar tips and the singlet region was measured to a mere $50 \mathrm{~nm}$ [20]. The discrepancies in the data might be explained by differences in dynamic states of the flagellum (growing compared with mature) or in sample preparation, as it was shown that chemical fixation induced depolymerisation of microtubules in the tips of T. brucei [27]. To settle this debate, HPF-FS or cryo-EM of C. reinhardtii mature flagella needs to be performed.

Model 3: Like in model 1, a central microtubule cap, complete with plates and bead, was observed in T. thermophila with three different EM techniques [22,28,29], except that its central pair microtubules extend approximately 200 $\mathrm{nm}$ longer than the A-tubules in the singlet region, giving the tip a more protruding shape. The bead of the central microtubule cap was not visible in the cryo-ET reconstructions of T. thermophila flagella tips [28]. Two other species of protist ciliates shared a similar tip ultrastructure [19,30].

Model 4: Four species of kinetoplastids including T. brucei, had no singlet region giving the tip a blunt rounded shape $[20,31]$. The central pair microtubules terminated approximately $50 \mathrm{~nm}$ farther from the tip compared with the doublets and in multiple instances B-tubules extended farther than the A-tubules of the same doublet $[18,27]$. Cryo-ET on mature T. brucei flagella confirmed the presence of carrot-like densities that protruded into the lumen of A-tubules also in this species [20].

Model 5: The singlet region in flagella of rodent and human spermatozoa extends for several micrometres and each singlet microtubule terminates at variable distance from the tip [32,33] (Zabeo and Höög, manuscript in preparation). In these systems the A- and B-tubules of doublet microtubules can split and form two complete singlet microtubules - a 'duplex'. In rodents, the singlets of each duplex microtubule were held together by electron-dense material at their distal tips, similar to the central pair microtubules in Model 2, and became structurally indistinguishable from the central pair itself [33]. However, the central pair could be tracked from the complete axoneme to the very tip of the flagellum, while not all duplex microtubules extended that far. Cryo-ET of human spermatozoa did not show any clear 


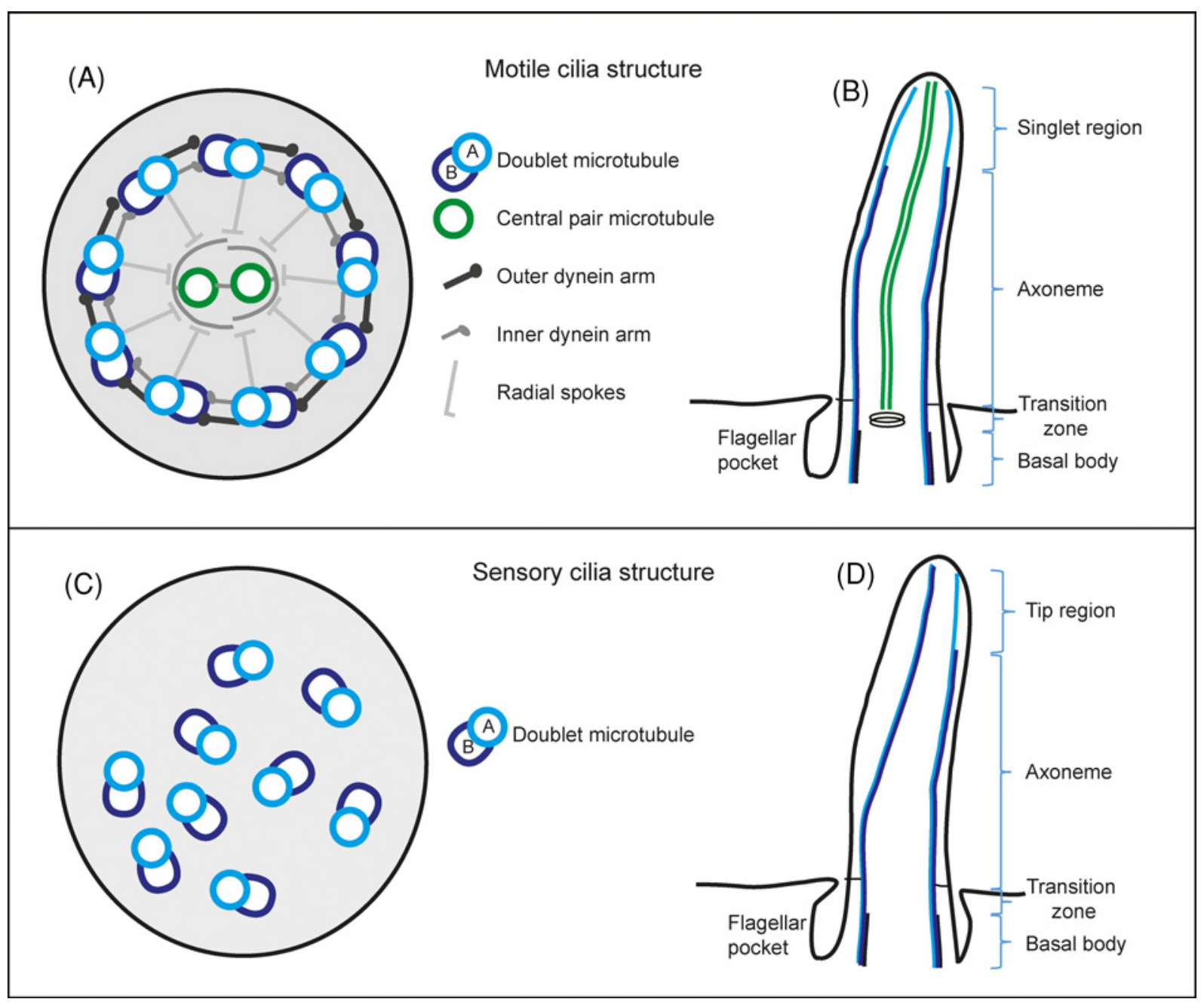

Figure 2. Schematic drawing of eukaryotic flagella ultrastructure

(A) Cross-sectional view of a motile cilium seen from the proximal end containing the canonical nine doublet microtubules and two central pair microtubules. (B) A motile cilium in longitudinal view showing the different regions along its length. (C) A cross-sectional view of a sensory cilium showing the variable orientation of the nine doublet microtubules and lack of central pair microtubules. (D) A sensory cilium and its different regions along its length.

connection between singlets of the same doublet after they split apart (Zabeo and Höög, manuscript in preparation). In this study, the central pair could not be traced along the entire singlet region but in one instance, it terminated before the start of the singlet region, $7.5 \mu \mathrm{m}$ from the flagellum tip. Densities associated with the distal ends of singlet microtubules, similar to the carrot-like plugs, were seen in chemically fixed rodent spermatozoa [33] and in cryo-ET of human spermatozoa (Zabeo and Höög, manuscript in preparation). In addition, an inner microtubule structure called TAILS, which spanned several micrometres, was discovered in the lumen of singlet, doublet and central pair microtubules in the human spermatozoon tip [32]. It was hypothesised that TAILS might prevent microtubule dynamicity or aid cell motility.

Model 6: Flagella from tracheal tissue of rabbit, chicken and bovine showed yet another tip ultrastructure, where only A-tubules and central pair microtubules extended into the singlet region and terminated at the tip by connecting to an electron-dense body that was called a ciliary cap [21]. Here, carrot-like densities seemed to anchor the distal ends of the singlet microtubules to the ciliary cap. Hair-like protrusions called the ciliary crown were found on the surface of the plasma membrane of the flagellar tip in rabbit and chicken, but not in bovine. The ciliary crown was still present after demembranation, suggesting that it might extend through the plasma membrane and be directly anchored to the ciliary cap. The A-tubules and central pair microtubules of flagella from gills of the freshwater mussel Elliptio complanatus also converge together at the tip where electron densities are associated with their distal ends, 


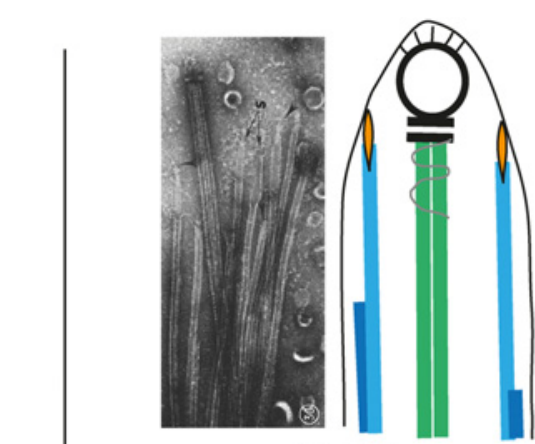

Model 1

Chlamydomonas reinhardtii (ref.17)

온

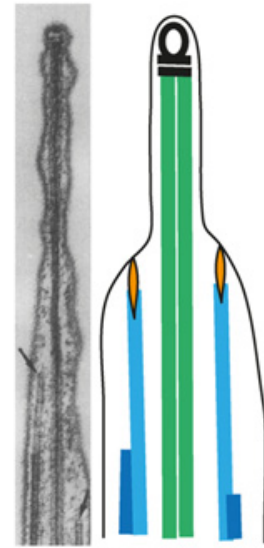

Model 3

Tetrahymena thermophila

Scourfieldia caeca

Spermatozopsis similis $(18,26,27,28,29)$

$\mid$

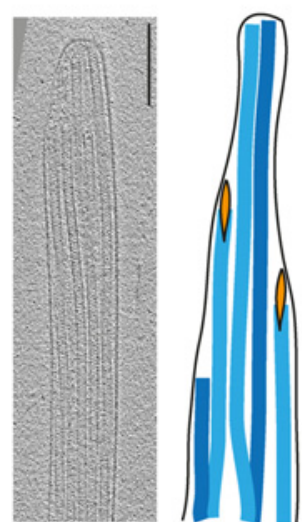

Model 5

Mesocricetus auratus Rattus norvegicus Homo sapiens Mus musculus $(31,32)$

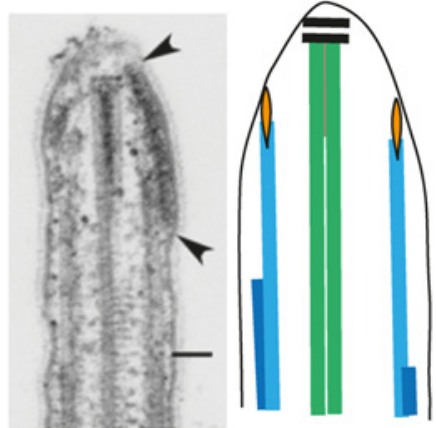

Model 2

Chlamydomonas reinhardti Aequipecten irridians $(19,20,21,22,23)$

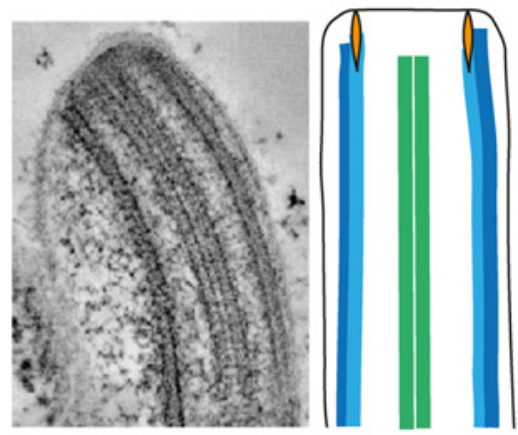

Model 4

Trypanosoma brucei, Leischmania major Crithidia deanei, Herpetomonas megaseliae $(24,30)$

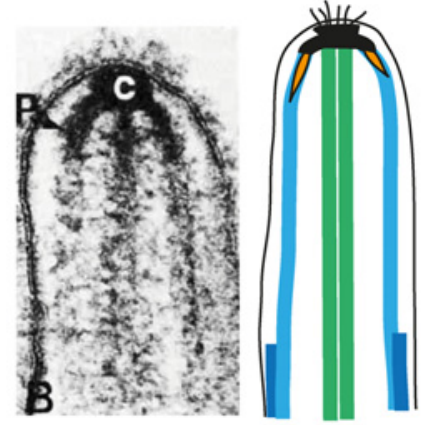

Model 6

Bos taurus, Beroe cucumis Oryctolagus cuniculus Gallus gallus domesticus Elliptio complanatus $(33,34,35)$

Figure 3. Schematic drawing illustrating six different models of motile flagellar structures published to date, and in which species each structure was shown

The A-tubules are shown in turquoise, B-tubules in blue, central pair microtubules in green, carrot-like plugs in orange and other electron-dense structures in black. Models are adjoined by original data reprinted with permission from publications: [17] for Model 1, [18] for Model 2, [19] for Model 3, [20] for Model 4 and [21] for Model 6. Cryo-EM in Model 5 is an original picture from the authors. All samples were prepared with chemical fixation or negative stain except for models 3 , 4, and 5 , that were shown with HPF-FS or cryo-EM too. Abbreviation: HPF-FS, high-pressure frozen and freeze substituted. 
(A)

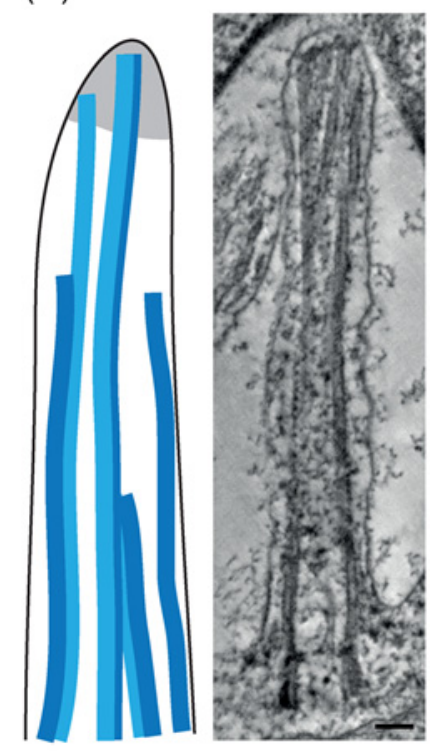

Canonical Sensory Cilium
(B)

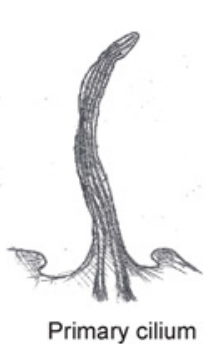

(E)

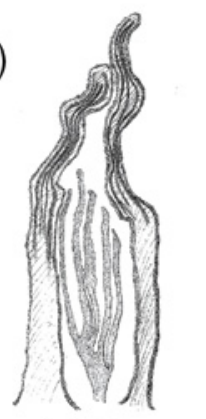

IL1 \& IL2
(C)

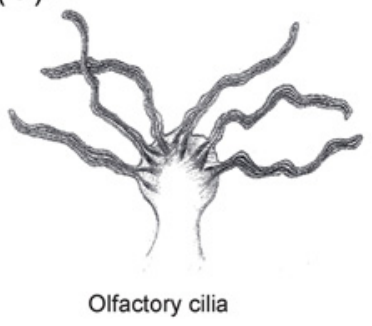

(F)

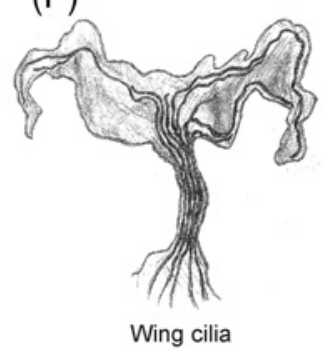

Wing cilia

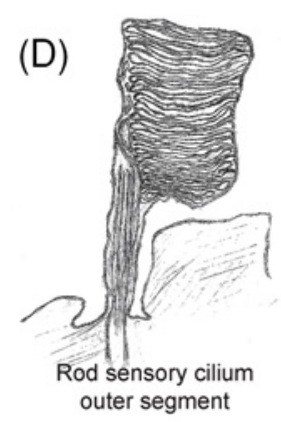

(G)

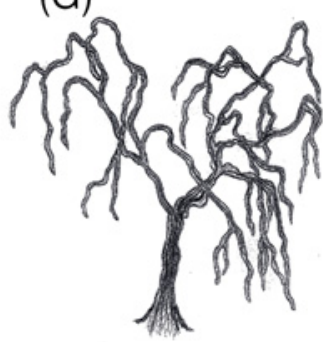

Tree-like cilia (AWA)

Figure 4. Divergence in the structure of sensory cilia tips

(A) A schematic drawing of a canonical sensory cilia tip, showing that both singlet and doublet microtubules can reach the distal most flagellar membrane. An electron density is found in the distal tip of some, but not all, sensory cilia. A tomographic slice from a $3 \mathrm{D}$ reconstruction of the entire length of the Leishmania mexicana amastigote flagellum. (Scale bar=100 $\mathrm{nm}$ ). (B-F) A gallery of the extreme modifications to structure that can be found in sensory cilia. The top row can be found in humans and animals whereas the bottom row are found in C. elegans neuronal sensory cilia.

however no ciliary crown was found [34]. Convergence to electron densities at the tip after a singlet region was also seen in the extreme structure of macrocilia in the ctenophore Beroe cucumis [35].

These different structures likely mirror different flagellar functions that are specific to each cell type and possibly influence flagellar stability and motility. Dentler and LeCluyse [21] speculated that a ciliary cap might provide a stronger stroke in flagella that beats in a more viscous environment, as in tracheal epithelia, compared with flagella that beat in water. They also suggested that the ciliary crown might help with mucus translocation, since it was only found in animal duct epithelia.

\section{Sensory cilia tip ultrastructure}

In contrast with motile cilia, sensory cilia are generally characterised by a $9+0$ axoneme which lacks central pair microtubules as well as inner and outer dynein arms. Consequently, sensory cilia are immotile and instead function like a cellular antenna to transduce optical, chemical, osmotic or mechanosensory signals [36]. Despite a resurgence of interest in the vertebrate primary cilium, the majority of information about its ultrastructure is from when chemical fixation techniques was the convention rather than the modern HPF-FS or cryo-EM [37-39]. Although microtubule doublets begin arranged in a circular symmetry in sensory cilia, similar to motile cilia, this $9+0$ arrangement is soon broken as one or two doublet microtubules folds in to form an $8+1$ or $7+2$ pattern $[37,40]$ which has been referred to as a 9 variable $(9 \mathrm{v})$ axoneme [41]. Near the tip, the arrangement of microtubules becomes more disordered, several doublet microtubules become singlets, and both doublet and singlet microtubules terminate as the cilium tapers resulting in a reduced number of microtubules that reach the tip (Figure 4A-B) $[18,41]$.

However, many sensory cilia have adopted extreme structures deviating from this theme as depicted in Figure 4. The axoneme of human olfactory cilia (Figure 4C) contains nine outer doublet microtubules with a variable number (2-4) of inner singlet microtubules [42]. Doublets are lost distally and the shaft tapers as microtubules terminate and only two or three singlet microtubules exist at the tip. Amphid channel cilia in the neurons of C. elegans also have between one and seven inner singlet microtubules composed of eleven protofilaments rather than the normal thirteen $[43,44]$. Near the tip, only singlet microtubules exist and the cilium tapers. 
The outer segment of rod sensory cilium in the eye contains a normal axonemal structure but the cilium is expanded to contain thousands of lamellar disk-shaped membranes containing rhodopsin and other proteins involved in phototransduction (Figure 4D) [45]. These disks decrease in size in the distal region of the flagellum, and are shed at the tip to be exocytosed by pigmented epithelial cells [46]. The eyes of the tardigrade Milnesium tardigradum also contain large branching, arborescent cilia that extend into their optic cavity [47].

Several other sensory neuronal cilia have been described using 3D EM of HPF-FS C. elegans worms, which have widely divergent axonemal and tip structures [44]. The function of such diversity is still largely unknown, but they may reflect specific needs for sensorial signalling, such as increasing the surface of receptor organelles. The tips of cilia in inner labia (IL1 and IL2) neurons contain four to six disorganised singlet microtubules, and curve sharply inwards before terminating. IL2 curves back outwards again at the tip (Figure 4E). Wing cilia flatten to form a large wing-like structure containing sparse singlet microtubules in their distal segments (Figure 4F). Successive and complex branching is observed in tree-like cilia, which can contain as many as 80 branches in which the nine microtubule doublets segregate haphazardly (Figure 4G). In the cilia of outer labia neurons (OLL and OLQ) and IL1, electron-dense material is detected at the tips. Furthermore, microtubules arranged in a square around a central filament comprise the tip of OLQ. Electron-dense material is also found surrounding microtubules at the cilium tip of the mechanosensory CEP neuron, which widens to form a cluster of many singlet microtubules that nucleate in this region. These recent studies have demonstrated that sensory cilia vary considerably both between and within organisms, and the power of modern EM is to visualise these complex structures in great detail.

\section{Is the distal tip a dynamic structure?}

The flagellum tip in C. reinhardtii is constantly growing and shrinking as seen by the incorporation of tagged tubulin at the axonemal microtubule plus ends [48]. This has led to a general understanding that eukaryotic flagella tips are dynamic structures. But how common is this dynamicity at the flagellum tip really? Neither T. brucei [49] nor mouse spermatozoa [50] have dynamic flagellar distal tips once they are fully grown. This was shown as their motile flagella remains the same length in the absence of intraflagellar transport (IFT), that delivers new building blocks to the tip [51]. It is interesting to note that dynamicity is not observed in flagellar models without a capping structure, like in $T$. brucei or mouse spermatozoa, while it exists in C. reinhardtii's capped flagella. However, data from a larger number of models need to be recorded before a correlation can be induced. The lack of dynamicity in spermatozoa end pieces agrees well with the hypothesis that the recently discovered TAILS complex is a microtubule stabiliser that prevents normal dynamic instability in the end piece in humans [32]. In contrast, C. elegans sensory cilia appear to maintain a dynamicity after formation, as it displays fluorescence recovery after photobleaching of tubulin-YFP [52] and the genes associated with ciliary assembly continue to be expressed even in adult worms [53]. Therefore, it appears that there are cases of both dynamic and non-dynamic distal tips in both motile and sensory cilia, making generalisations difficult.

\section{Proteins residing at the cilium tip}

Compared with the axoneme and the basal body, relatively little is known about the biochemical composition of ciliary tips. Recently, over 20 proteins that reside in ciliary tips have been identified and we have gathered them into a list (Table 1). These are involved in functions including, but not limited to, ciliary dynamics, contribution to the tip ultrastructure, regulation of IFT events and signalling.

\section{Proteins involved in regulating ciliary dynamics}

End binding proteins (EBs) are a widely conserved family of proteins involved in regulating microtubule dynamics, localising mainly to the dynamic plus ends of microtubules throughout the cell [80]. EB1 also localises to the tips of microtubules in C. reinhardtii cilia, and human EB3 has been found at the tips of primary cilia in bronchial epithelial cells $[54,55]$. The mammalian protein CEP104 (homologue in C. reinhardtii, FAP256) is localised to the cilium tip in an EB-dependent manner [69] and appears to play a vital role in ciliogenesis. CEP104 relocates from the distal ends of the centriole to the ciliary tips as ciliogenesis begins [25]. Cells depleted of CEP104/FAP256 display complete ciliary loss or short, blunt cilia, due to doublet microtubules of the same length as the central pair microtubules [25]. Furthermore, CEP104/FAP256 contains a tubulin-binding TOG domain present in other proteins that regulate microtubule dynamics [69].

Various members of the kinesin superfamily (Kinesin-13, Kif7, Kif19A) regulate ciliary length at the tips by affecting microtubule polymerisation dynamics, and consequently cells deficient in certain KIFs display abnormally long and unstable cilia [61,63-65]. In C. reinhardtii however, kinesin-13 appears to be absent from flagellar tips but 
Table 1 Protein residents of the flagellum tip identified by immunofluorescence or immunogold labelling in various organisms

\begin{tabular}{|c|c|c|c|c|c|c|c|c|c|c|c|c|}
\hline Protein & Involved In & Tb & $\mathrm{Cr}$ & Hs & Mm & $\mathrm{Ce}$ & Dm & Gi & Tt & Lm & $\mathbf{X I}$ & Ref. \\
\hline EB1 & Dynamics, IFT regulation & & & & & & & & & & & [54-58] \\
\hline EB3 & Dynamics & & & & & & & & & & & {$[55,59]$} \\
\hline CDPK1 & IFT regulation & & & & & & & & & & & [60] \\
\hline FLA8 (Kif3B) & IFT regulation & & & & & & & & & & & [60] \\
\hline Kif19A & Dynamics & & & & & & & & & & & {$[61,62]$} \\
\hline Kinesin 13 & Dynamics & & & & & & & 6 & & $(8 \infty)$ & & {$[63,64,58]$} \\
\hline Kif7 & Dynamics, signalling & & & & & & & & & & & [65-67] \\
\hline Kif17 (OSM-3) & IFT motor & & & & & $\because \%$ & & & & & & [68] \\
\hline $\begin{array}{l}\text { CEP104 (CrFAP256, } \\
\text { TbACS3) }\end{array}$ & Ciliogenesis & & & & & & & & & & & {$[25,69,70]$} \\
\hline PRMT1 & Dynamics & & & & & & & & & & & [71] \\
\hline PRMT3 & Dynamics & & & & & & & & & & & [71] \\
\hline PRMT5 & Dynamics & & & & & & & & & & & [71] \\
\hline PRMT10 & Dynamics & & & & & & & & & & & [71] \\
\hline Agglutinin & Tip adhesion & & & & & & & & & & & [72] \\
\hline ACS1 & Structure & & & & & & & & & & & [70] \\
\hline ACS2 & Structure & & & & & & & & & & & [70] \\
\hline FCP1 & Structure & & & & & & & & & & & [70] \\
\hline FCP4 (TbKin15) & Structure & & & & & & & & & & & [70] \\
\hline Sentan & Structure & & & & & & & & & & & [73] \\
\hline Amo & Sperm activation & & & & & & & & & & & {$[74,75]$} \\
\hline NOMPC & Signalling & & & & & & & & & & & [76] \\
\hline Gli2 & Signalling & & & & & & & & & & & {$[67,77,78]$} \\
\hline Gli3 & Signalling & & & & & & & & & & & {$[67,77,78]$} \\
\hline Sufu & Signalling & & & & & & & & & & & {$[65,78]$} \\
\hline
\end{tabular}

functions to depolymerise cytoplasmic microtubules in order to generate a pool of tubulin subunits during flagellar regeneration. In addition to these known microtubule-associated proteins, four protein arginine methyltransferases localise to the tip of C. reinhardtii cilia where they play a role in regulating resorption of the flagellum [71]. 
Table 1 Protein residents of the flagellum tip identified by immunofluorescence or immunogold labelling in various organisms (Continued)

\begin{tabular}{|c|c|c|c|c|c|c|c|c|c|c|c|c|}
\hline Protein & Involved In & Tb & $\mathrm{Cr}$ & Hs & Mm & $\mathrm{Ce}$ & Dm & Gi & Tt & Lm & $\mathbf{X I}$ & Ref. \\
\hline DCDC2C & Unknown & & & & & & & & & & & [12] \\
\hline Tb927.1.2710 & Unknown & & & & & & & & & & & [70] \\
\hline FLAM8 & Unknown & & & & & & & & & & & [11] \\
\hline CALP1.3 & Unknown & & & & & & & & & & & [79] \\
\hline SKCRP7.2 & Unknown & & & & & & & & & & & [79] \\
\hline $\begin{array}{l}\text { Blue cross se } \\
\text { Chlamydomo } \\
\text { Mm, Mus mu }\end{array}$ & $\begin{array}{l}\text { cilium indicat } \\
\text { rdtii; Dm, Dros } \\
\text { fu, suppressor }\end{array}$ & & $\begin{array}{l}\text { gree } \\
\text { B, en } \\
\text { ma b }\end{array}$ & dicat & $\begin{array}{l}\text { resen } \\
\text { tein; } \\
\text { rahym }\end{array}$ & in ser & $\begin{array}{l}\text { y cilia. } \\
\text { ntestina } \\
\text { phila; } X\end{array}$ & $\begin{array}{l}\text { prev } \\
\text {; Hs, } \\
\text { Xeno }\end{array}$ & ns: & & $\begin{array}{l}\text { bditis } \\
\text { eish }\end{array}$ & $\begin{array}{l}\text { yans; } 0 \\
\text { a majo }\end{array}$ \\
\hline
\end{tabular}

\section{IFT turnaround is regulated at the tip}

Anterograde IFT trains deliver building blocks to the ciliary assembly site in the distal tip. There the IFT trains are reloaded with different cargo and then moved in the retrograde direction - towards the cell body. Therefore, the ciliary tip is an important site of IFT regulation and components of IFT trains will transiently localise to this area (reviewed by Taschner and Lorentzen [81]). Here we will focus on the few tip resident proteins that have been found to be involved in IFT transport. In C. elegans, many cilia contain extended singlet zones where kinesin-2 (which carries IFT cargo the whole length of flagella in C. reinhardtii) disassociates. Instead, the motor protein OSM-3 functions to extend the singlet zone by carrying IFT along singlet microtubules to the tip [68]. In C. reinhardtii, the kinase CDPK1 (CrCDPK1) relocates from the proximal cilium to the tip where it regulates IFT turnaround during flagellar assembly. CrCDPK1 is thought to phosphorylate FLA8, another protein that accumulates at the tip of the growing flagellum, which then causes dissociation of anterograde IFT-B train from its kinesin-2 motor [60]. Furthermore, IFT172, a component of IFT-B, is required for transition between anterograde and retrograde IFT and has been suggested to do so by interaction with EB1 upon arrival at the tip $[82,56]$. Further details of the molecular mechanisms underpinning IFT turnaround will be exciting advancements in years to come.

Together, our current knowledge of the cilium tip proteome suggests that the protein composition of the flagellum tip is variable between species. It remains to be seen if a core set of proteins emerge as conserved cilium-tip residents as additional proteomics data and localizstions become available in the future. One such promising advancement is the whole proteome of T. brucei being localised in the TrypTag project [83]. With that we will have a first complete list of proteins that localise to the flagellum tip in one species.

\section{The primary cilium tip and signalling}

In recent years, the primary cilium has emerged as a major hub for signal transduction pathways including Hedgehog (Hh), Wnt and PDGFR $\alpha$ signalling [4]. Perhaps the best-characterised signalling pathway that critically depends on the primary cilium is the vertebrate Hh signal transduction, which is disrupted in a number of tumors and important for embryonic development and adult tissue homoeostasis [5,6]. It is observed that the axoneme-associated protein complex of the Hh pathway, which is composed of the kinesin Kif7, the Gli transcription factors and their negative regulator Suppressor of fused (Sufu), localises to a submicron region at the distal tip of the primary cilium upon pathway activation (Figure 5) $[65,66,67,77]$. In current models, it is suggested that Gli is activated at the cilium tip, and subsequently transported to the nucleus for gene activation [84]. These observations raise some fundamental questions: What is the mechanism by which Kif7, Gli and Sufu proteins localise to the cilium tip upon pathway activation? What is the functional consequence of concentrating these proteins at the cilium tip?

Initial clues to these questions come from studies on Kif7, a conserved protein of the Hh signalling pathway [85]. Mutations in this ciliary kinesin are associated with a number of ciliopathies [85]. In the absence of Kif7, Gli can enter the cilium but is not concentrated at the cilium tip [65,77]. These initial findings suggested that Kif7 might be a plus-end directed transport motor that transports Gli to the cilium tip. However, in vitro studies indicate that Kif7 is a non-motile motor that autonomously recognises the plus-ends of microtubules and suppresses microtubule polymerisation rates $[65,86]$. Consistent with these observations, the knockdown of Kif7 results in an over-elongated 


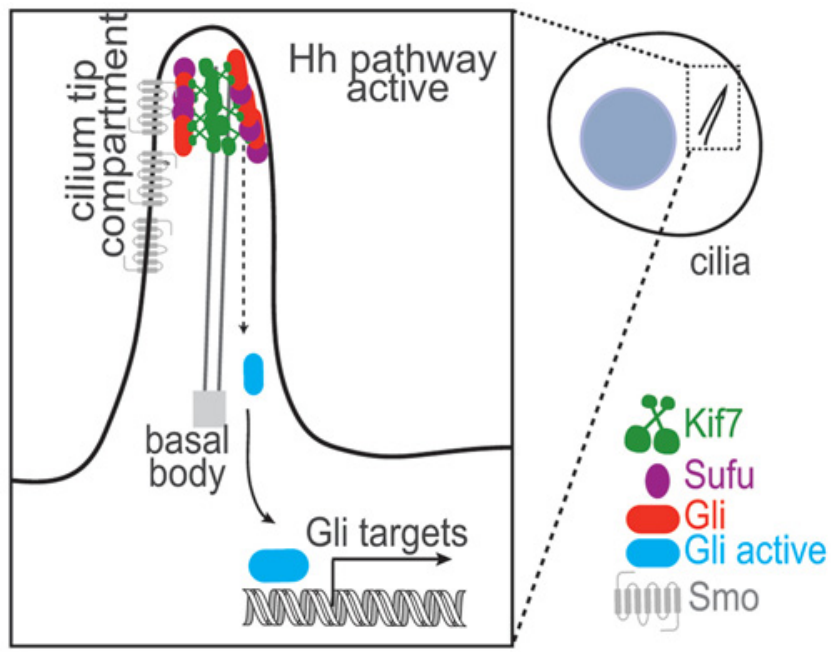

Figure 5. Schematic representation shows the localisation of the kinesin Kif7, the transcription factor Gli and its repressor Sufu to the distal cilium-tip upon Hh pathway activation

Gli is activated at the cilium tip and subsequently translocates to the nucleus.

cilium with defective architecture [65]. Together, these cell biological and biochemical studies have led to the proposal that Kif7 plays a role in sculpting the cilium tip-architecture to establish a single 'compartment' for the proper recruitment and regulation of Gli and Sufu. Currently the mechanism by which Kif7 organises such a platform at the distal cilium tip is unknown. Furthermore, whether Kif7 plays additional roles in Gli activation besides localising it to the cilium tip is unclear. How membrane-associated proteins modulate the specification of a tip compartment at the distal end of the axoneme remains an open question. Finally, it remains to be elucidated if the distal cilium tip acts as a compartment for biochemical reactions underlying other cilium-dependent signalling pathways.

\section{Signalling in motile cilia}

Primary cilia are well known for possessing sensorial and signalling functions in almost all animals [87]. However, sensorial functions of both mechanoreceptive and chemoreceptive nature have been reported for motile cilia in mammals [88-92] and protists [93,94], as reviewed previously [95]. Most of these functions have not been specifically associated with the cilium tip, but rather with the whole organelle. In some instances, primary cilium tips have been observed making direct contact with a nearby cell or organelle, which might underlie signalling processes as well [96].

The tip of motile cilia is reported to have signalling functions in C. reinhardtii mating. Solter and Gibor [94] first observed that deflagellated cells could not initiate mating. Agglutinins, long and mating type-specific glycoproteins [97], allow mating gametes to come in contact. Within seconds, a process termed as flagellar tip activation [98] occurs: densities accumulate at the flagellar tip, between the outer doublet microtubules and the plasma membrane, and the microtubules in the singlet region polymerise. This causes the tip to swell and increase in length by approximately $30 \%$ of its original size. Both in C. reinhardtii and C. eugametos, agglutinins accumulate at the flagellum tip [99-101] and likely contribute to its swelling. The flagellar tip activation was shown to rise cAMP levels [102] and activate protein tyrosine kinase activity [103].

Motile cilia are also known to release extracellular vesicles from the plasma membrane (ectosomes), which can deliver signals or biomolecules to target cells [104]. A recent review in this journal covered this topic extensively [105]. In C. reinhardtii the release of ectosomes localises at the flagellum tip [100,106].

\section{Conclusion}

The eukaryotic flagellum distal tip is the place of ciliary growth and a hub for cellular signalling. In this review we show that the motile flagella distal tip structures described to date could be sorted into six different models. Primary cilia also show a wide array of tip structures, showing a widespread evolutionary adaptability of this region and a difficulty to generalise from research on a few model organisms only. In the past 15 years, 29 protein residents of the flagellar tip have been identified in different organisms, but a complete flagellar tip proteome from any one species is still 
missing. Knowing all the structure and components found in this region would greatly increase our understanding of the processes of ciliogenesis and signalling emanating from the flagellum tip.

\section{Summary}

- Both motile and primary cilia have a wide diversity of structures at the distal tip.

- A list of the proteins known to reside at the distal tip is presented.

- The distal tip is a hub of cellular signalling both in motile and primary cilia.

\section{Acknowledgements}

We thank Dimitra Panagaki for contributing with beautiful artwork of primary cilia structures and Per Widlund for providing comments on the manuscript.

\section{Funding}

This work was supported by the Swedish Research Council Young Investigator Grant [grant number 2015-05427 (to J.L.H.)]; the Pew Charitable Trust (to R.S.) and the Smith Family Foundation (to R.S.).

\section{Competing interests}

The authors declare that there are no competing interests associated with the manuscript.

\section{Abbreviations}

EB, end binding protein; EM, electron microscopy; ET, electron tomography; Hh, Hedgehog; HPF-FS, high-pressure frozen and freeze substituted; IFT, intraflagellar transport; KIF, kinesin superfamily protein; OSM-3, osmotic avoidance abnormal protein 3; PDGFR, platelet-derived growth factor; Sufu, suppressor of fused; TAILS, tail axoneme intra-lumenal spiral; Wnt, Wingless/integrated.

\section{References}

1 Khan, S. and Scholey, J.M. (2018) Assembly, functions and evolution of archaella, flagella and cilia. Curr. Biol. 28, R278-R292, https://doi.org/10.1016/j.cub.2018.01.085

2 Sherwin, T. (1987) Distinct localization and cell cycle dependence of $\mathrm{COOH}$ terminally tyrosinolated alpha-tubulin in the microtubules of Trypanosoma brucei brucei. J. Cell Biol. 104, 439-446, https://doi.org/10.1083/jcb.104.3.439

3 Sherwin, T. and Gull, K. (1989) Visualization of detyrosination along single microtubules reveals novel mechanisms of assembly during cytoskeletal duplication in trypanosomes. Cell 57, 211-221, https://doi.org/10.1016/0092-8674(89)90959-8

4 Johnson, K.A. (1992) Polarity of flagellar assembly in Chlamydomonas. J. Cell Biol. 119, 1605-1611, https://doi.org/10.1083/jcb.119.6.1605

5 Wheway, G., Nazlamova, L. and Hancock, J.T. (2018) Signaling through the primary cilium. Front Cell Dev Biol. 6, 8, https://doi.org/10.3389/fcell.2018.00008

6 Briscoe, J. and Therond, P.P. (2013) The mechanisms of Hedgehog signalling and its roles in development and disease. Nat. Rev. Mol. Cell Biol. 14, 416-429, https://doi.org/10.1038/nrm3598

7 Goetz, S.C. and Anderson, K.V. (2010) The primary cilium: a signalling centre during vertebrate development. Nat. Rev. Genet. 11, 331-344, https://doi.org/10.1038/nrg2774

8 Reiter, J.F. and Leroux, M.R. (2017) Genes and molecular pathways underpinning ciliopathies. Nat. Rev. Mol. Cell Biol. 18, 533-547, https://doi.org/10.1038/nrm.2017.60

9 Nicastro, D., Fu, X., Heuser, T., Tso, A., Porter, M.E. and Linck, R.W. (2011) Cryo-electron tomography reveals conserved features of doublet microtubules in flagella. Proc. Natl. Acad. Sci. U.S.A. 108, E845-E853, https://doi.org/10.1073/pnas.1106178108

10 Loreng, T.D. and Smith, E.F. (2017) The central apparatus of cilia and eukaryotic flagella. Cold Spring Harb. Perspect Biol. 9, a028118, https://doi.org/10.1101/cshperspect.a028118

11 Subota, I., Julkowska, D., Vincensini, L., Reeg, N., Buisson, J., Blisnick, T. et al. (2014) Proteomic analysis of intact flagella of procyclic Trypanosoma brucei cells identifies novel flagellar proteins with unique sub-localization and dynamics. Mol. Cell. Proteomics 13, 1769-1786, https://doi.org/10.1074/mcp.M113.033357

12 Jumeau, F., Chalmel, F., Fernandez-Gomez, F.-J., Carpentier, C., Obriot, H., Tardivel, M. et al. (2017) Defining the human sperm microtubulome: an integrated genomics approach. Biol. Reprod. 96, 93-106

13 Pazour, G.J., Agrin, N., Leszyk, J. and Witman, G.B. (2005) Proteomic analysis of a eukaryotic cilium. J. Cell Biol. 170, 103-113, https://doi.org/10.1083/jcb.200504008 
14 Mayer, U., Küller, A., Daiber, P.C., Neudorf, I., Warnken, U., Schnölzer, M. et al. (2009) The proteome of rat olfactory sensory cilia. Proteomics 9 , 322-334, https://doi.org/10.1002/pmic.200800149

15 Ishikawa, H., Thompson, J., Yates, J.R. and Marshall, W.F. (2012) Proteomic analysis of mammalian primary cilia. Curr. Biol. 22, 414-419, https://doi.org/10.1016/j.cub.2012.01.031

16 Fisch, C. and Dupuis-Williams, P. (2011) Ultrastructure of cilia and flagella - back to the future. Biol Cell. 103, 249-270, https://doi.org/10.1042/BC20100139

17 Dentler, W.L. and Rosenbaum, J.L. (1977) Flagellar elongation and shortening in Chlamydomonas. III. structures attached to the tips of flagellar microtubules and their relationship to the directionality of flagellar microtubule assembly. J. Cell Biol. 74, 747-759

18 Rogowski, M., Scholz, D. and Geimer, S. (2013) Electron microscopy of flagella, primary cilia, and intraflagellar transport in flat-embedded cells. Methods Enzymol. 524, 243-263, https://doi.org/10.1016/B978-0-12-397945-2.00014-7

19 Melkonian, M. and Preisig, H.R. (1982) Twist of central pair microtubules in the flagellum of the green flagellate Scourfieldia caeca. Cell Biol. Int. Rep. 6, 269-277, https://doi.org/10.1016/0309-1651(82)90079-0

20 Höög, J.L., Lacomble, S., O’Toole, E.T., Hoenger, A., McIntosh, J.R. and Gull, K. (2014) Modes of flagellar assembly in Chlamydomonas reinhardtii and Trypanosoma brucei. eLife. 3, e01479, https://doi.org/10.7554/eLife.01479

21 Dentler, W.L. and LeCluyse, E.L. (1982) The effects of structures attached to the tips of tracheal ciliary microtubules on the nucleation of microtubule assembly in vitro. Cell Motil. 2, 13-18, https://doi.org/10.1002/cm.970020705

22 Dentler, W.L. (1980) Structures linking the tips of ciliary and flagellar microtubules to the membrane. J. Cell Sci. 42, 207-220

23 Ringo, D.L. (1967) Flagellar motion and fine structure of the flagellar apparatus in Chlamydomonas. J. Cell Biol. 33, 543-571, https://doi.org/10.1083/jcb.33.3.543

24 Dentler, W. (2005) Intraflagellar transport (IFT) during assembly and disassembly of Chlamydomonas flagella. J. Cell Biol. 170, 649-659, https://doi.org/10.1083/jcb.200412021

25 Satish Tammana, T.V., Tammana, D., Diener, D.R. and Rosenbaum, J. (2013) Centrosomal protein CEP104 (Chlamydomonas FAP256) moves to the ciliary tip during ciliary assembly. J. Cell Sci. 126, 5018-5029, https://doi.org/10.1242/jcs.133439

26 Vannuccini, E., Paccagnini, E., Cantele, F., Gentile, M., Dini, D., Fino, F. et al. (2016) Two classes of short intraflagellar transport train with different 3D structures are present in Chlamydomonas flagella. J. Cell Sci. 129, 2064-2074, https://doi.org/10.1242/jcs.183244

27 Höög, J.L., Lacomble, S., Bouchet-Marquis, C., Briggs, L., Park, K., Hoenger, A. et al. (2016) 3D architecture of the Trypanosoma brucei flagella connector, a mobile transmembrane junction. PLoS Negl. Trop Dis. 10, e0004312, https://doi.org/10.1371/journal.pntd.0004312

28 Dentler, W.L. (1984) Attachment of the cap to the central microtubules of Tetrahymena cilia. J. Cell Sci. 66, 167-173

29 Reynolds, M.J., Phetruen, T., Fisher, R.L., Chen, K., Pentecost, B.T., Gomez, G. et al. (2018) The developmental process of the growing motile ciliary tip region. Sci. Rep. 8, 7977, https://doi.org/10.1038/s41598-018-26111-2

30 Melkonian, M. and Preisig, H.R. (1984) Ultrastructure of the flagellar apparatus in the green flagellate Spermatozopsis similis. Plant Syst. Evol. 146, 145-162, https://doi.org/10.1007/BF00989542

31 Woolley, D., Gadelha, C. and Gull, K. (2006) Evidence for a sliding-resistance at the tip of the trypanosome flagellum. Cell Motil. Cytoskeleton 63 , 741-746, https://doi.org/10.1002/cm.20159

32 Zabeo, D., Heumann, J.M., Schwartz, C.L., Suzuki-Shinjo, A., Morgan, G., Widlund, P.O. et al. (2018) A lumenal interrupted helix in human sperm tail microtubules. Sci. Rep. 8, https://doi.org/10.1038/s41598-018-21165-8

33 Woolley, D.M. and Nickels, S.N. (1985) Microtubule termination patterns in mammalian sperm flagella. J. Ultrastruct. Res. 90, 221-234, https://doi.org/10.1016/S0022-5320(85)80001-0

34 Satir, P. (1968) Studies on cilia. Further studies on the cilium tip and a "sliding filament" model of ciliary motility. J. Cell Biol. 39, 77-94, https://doi.org/10.1083/jcb.39.1.77

35 Tamm, S.L. and Tamm, S. (1985) Visualization of changes in ciliary tip configuration caused by sliding displacement of microtubules in macrocilia of the ctenophore Beroe. J. Cell Sci. 79, 161-179

36 Falk, N., Lösl, M., Schröder, N. and Gießl, A. (2015) Specialized cilia in mammalian sensory systems. Cells 4, 500-519, https://doi.org/10.3390/cells4030500

37 Dahl, H.A. (1963) Fine structure of cilia in rat cerebral cortex. Z. Für. Zellforsch Mikrosk Anat. 60, 369-386, https://doi.org/10.1007/BF00336612

38 Allen, R.A. (1965) Isolated cilia in inner retinal neurons and in retinal pigment epithelium. J. Ultrastruct. Res. $12,730-747$, https://doi.org/10.1016/S0022-5320(65)80058-2

39 Yamamoto, M. and Kataoka, K. (1986) Electron microscopic observation of the primary cilium in the pancreatic islets. Arch. Histol. Jpn. 49, 449-457

40 Wilsman, N.J., Farnum, C.E. and Reed-Aksamit, D.K. (1980) Incidence and morphology of equine and murine chondrocytic cilia. Anat. Rec. 197, 355-361, https://doi.org/10.1002/ar.1091970309

41 Gluenz, E., Höög, J.L., Smith, A.E., Dawe, H.R., Shaw, M.K. and Gull, K. (2010) Beyond 9+0: noncanonical axoneme structures characterize sensory cilia from protists to humans. FASEB J. 24, 3117-3121, https://doi.org/10.1096/fj.09-151381

42 Moran, D.T., Rowley, J.C., Jafek, B.W. and Lovell, M.A. (1982) The fine structure of the olfactory mucosa in man. J. Neurocytol. 11, 721-746, https://doi.org/10.1007/BF01153516

43 Perkins, L.A., Hedgecock, E.M., Thomson, J.N. and Culotti, J.G. (1986) Mutant sensory cilia in the nematode Caenorhabditis elegans. Dev. Biol. 117, 456-487, https://doi.org/10.1016/0012-1606(86)90314-3

44 Doroquez, D.B., Berciu, C., Anderson, J.R., Sengupta, P. and Nicastro, D. (2014) A high-resolution morphological and ultrastructural map of anterior sensory cilia and glia in Caenorhabditis elegans. eLife 3, e01948, https://doi.org/10.7554/eLife.01948

45 Gilliam, J.C., Chang, J.T., Sandoval, I.M., Zhang, Y., Li, T., Pittler, S.J. et al. (2012) Three-dimensional architecture of the rod sensory cilium and its disruption in retinal neurodegeneration. Cell 151, 1029-1041, https://doi.org/10.1016/j.cell.2012.10.038 
46 Nickell, S., Park, PS-H, Baumeister, W. and Palczewski, K. (2007) Three-dimensional architecture of murine rod outer segments determined by cryoelectron tomography. J. Cell Biol. 177, 917-925, https://doi.org/10.1083/jcb.200612010

47 Greven, H. (2007) Comments on the eyes of tardigrades. Arthropod Struct. Dev. 36, 401-407, https://doi.org/10.1016/j.asd.2007.06.003

48 Marshall, W.F. and Rosenbaum, J.L. (2001) Intraflagellar transport balances continuous turnover of outer doublet microtubules: implications for flagellar length control. J. Cell Biol. 155, 405-414, https://doi.org/10.1083/jcb.200106141

49 Fort, C., Bonnefoy, S., Kohl, L. and Bastin, P. (2016) Intraflagellar transport is required for the maintenance of the trypanosome flagellum composition but not its length. J. Cell Sci. 129, 3026-3041, https://doi.org/10.1242/jcs.188227

50 San Agustin, J.T., Pazour, G.J. and Witman, G.B. (2015) Intraflagellar transport is essential for mammalian spermiogenesis but is absent in mature sperm. Mol. Biol. Cell 26, 4358-4372, https://doi.org/10.1091/mbc.e15-08-0578

51 Kozminski, K.G., Johnson, K.A., Forscher, P. and Rosenbaum, J.L. (1993) A motility in the eukaryotic flagellum unrelated to flagellar beating. Proc. Natl. Acad. Sci. U.S.A. 90, 5519-5523, https://doi.org/10.1073/pnas.90.12.5519

52 Hao, L., Thein, M., Brust-Mascher, I., Civelekoglu-Scholey, G., Lu, Y., Acar, S. et al. (2011) Intraflagellar transport delivers tubulin isotypes to sensory cilium middle and distal segments. Nat. Cell Biol. 13, 790-798, https://doi.org/10.1038/ncb2268

53 Fujiwara, M., Ishihara, T. and Katsura, I. (1999) A novel WD40 protein, CHE-2, acts cell-autonomously in the formation of $C$. elegans sensory cilia. Dev. Camb. Engl. 126, 4839-4848

54 Harris, J.A., Liu, Y., Yang, P., Kner, P. and Lechtreck, K.F. (2016) Single-particle imaging reveals intraflagellar transport-independent transport and accumulation of EB1 in Chlamydomonas flagella. Mol. Biol. Cell 27, 295-307, https://doi.org/10.1091/mbc.e15-08-0608

55 Schroder, J.M., Larsen, J., Komarova, Y., Akhmanova, A., Thorsteinsson, R.I., Grigoriev, I. et al. (2011) EB1 and EB3 promote cilia biogenesis by several centrosome-related mechanisms. J. Cell Sci. 124, 2539-2551, https://doi.org/10.1242/jcs.085852

56 Pedersen, L.B., Miller, M.S., Geimer, S., Leitch, J.M., Rosenbaum, J.L. and Cole, D.G. (2005) Chlamydomonas IFT172 is encoded by FLA11, interacts with CrEB1, and regulates IFT at the flagellar tip. Curr. Biol. 15, 262-266, https://doi.org/10.1016/j.cub.2005.01.037

57 Pedersen, L.B., Geimer, S., Sloboda, R.D. and Rosenbaum, J.L. (2003) The Microtubule Plus End-Tracking protein EB1 is localized to the flagellar tip and basal bodies in Chlamydomonas reinhardtii. Curr. Biol. 13, 1969-1974, https://doi.org/10.1016/j.cub.2003.10.058

58 Dawson, S.C., Sagolla, M.S., Mancuso, J.J., Woessner, D.J., House, S.A., Fritz-Laylin, L. et al. (2007) Kinesin-13 regulates flagellar, interphase, and mitotic microtubule dynamics in Giardia intestinalis. Eukaryot. Cell 6, 2354-2364, https://doi.org/10.1128/EC.00128-07

59 Brooks, E.R. and Wallingford, J.B. (2012) Control of vertebrate intraflagellar transport by the planar cell polarity effector Fuz. J. Cell Biol. 198, 37-45, https://doi.org/10.1083/jcb.201204072

60 Liang, Y., Pang, Y., Wu, Q., Hu, Z., Han, X., Xu, Y. et al. (2014) FLA8/KIF3B phosphorylation regulates kinesin-Il interaction with IFT-B to control IFT entry and turnaround. Dev. Cell 30, 585-597, https://doi.org/10.1016/j.devcel.2014.07.019

61 Niwa, S., Nakajima, K., Miki, H., Minato, Y., Wang, D. and Hirokawa, N. (2012) KIF19A is a microtubule-depolymerizing kinesin for ciliary length control. Dev. Cell 23, 1167-1175, https://doi.org/10.1016/j.devcel.2012.10.016

62 Wang, D., Nitta, R., Morikawa, M., Yajima, H., Inoue, S., Shigematsu, H. et al. (2016) Motility and microtubule depolymerization mechanisms of the Kinesin-8 motor, KIF19A. eLife 5, e18101, https://doi.org/10.7554/eLife.18101

63 Blaineau, C., Tessier, M., Dubessay, P., Tasse, L., Crobu, L., Pagès, M. et al. (2007) A novel microtubule-depolymerizing kinesin involved in length control of a eukaryotic flagellum. Curr. Biol. 17, 778-782, https://doi.org/10.1016/j.cub.2007.03.048

64 Chan, K.Y. and Ersfeld, K. (2010) The role of the Kinesin-13 family protein TbKif13-2 in flagellar length control of Trypanosoma brucei. Mol. Biochem. Parasitol. 174, 137-140, https://doi.org/10.1016/j.molbiopara.2010.08.001

65 He, M., Subramanian, R., Bangs, F., Omelchenko, T., Liem Jr, K.F., Kapoor, T.M. et al. (2014) The kinesin-4 protein Kif7 regulates mammalian Hedgehog signalling by organizing the cilium tip compartment. Nat. Cell Biol. 16, 663-672, https://doi.org/10.1038/ncb2988

66 Liem, K.F.J., He, M., Ocbina, P.J.R. and Anderson, K.V. (2009) Mouse Kif7/Costal2 is a cilia-associated protein that regulates Sonic hedgehog signaling. Proc. Natl. Acad. Sci. U.S.A. 106, 13377-13382, https://doi.org/10.1073/pnas.0906944106

67 Endoh-Yamagami, S., Evangelista, M., Wilson, D., Wen, X., Theunissen, J.-W., Phamluong, K. et al. (2009) The mammalian Cos2 homolog Kif7 plays an essential role in modulating Hh signal transduction during development. Curr. Biol. 19, 1320-1326, https://doi.org/10.1016/j.cub.2009.06.046

68 Evans, J.E., Snow, J.J., Gunnarson, A.L., Ou, G., Stahlberg, H., McDonald, K.L. et al. (2006) Functional modulation of IFT kinesins extends the sensory repertoire of ciliated neurons in Caenorhabditis elegans. J. Cell Biol. 172, 663-669, https://doi.org/10.1083/jcb.200509115

69 Rezabkova, L., Kraatz, S.H.W., Akhmanova, A., Steinmetz, M.0. and Kammerer, R.A. (2016) Biophysical and structural characterization of the centriolar protein Cep104 interaction network. J. Biol. Chem. 291, 18496-18504, https://doi.org/10.1074/jbc.M116.739771

70 Varga, V., Moreira-Leite, F., Portman, N. and Gull, K. (2017) Protein diversity in discrete structures at the distal tip of the trypanosome flagellum. Proc. Natl. Acad. Sci. U.S.A. 114, E6546-55, https://doi.org/10.1073/pnas.1703553114

71 Mizuno, K. and Sloboda, R.D. (2017) Protein arginine methyltransferases interact with intraflagellar transport particles and change location during flagellar growth and resorption. Mol. Biol. Cell 28, 1208-1222, https://doi.org/10.1091/mbc.e16-11-0774

72 Goodenough, U.W. (1993) Tipping of flagellar agglutinins by gametes of Chlamydomonas reinhardtii. Cell Motil. Cytoskeleton 25, 179-189, https://doi.org/10.1002/cm.970250207

73 Kubo, A., Yuba-Kubo, A., Tsukita, S., Tsukita, S. and Amagai, M. (2008) Sentan: a novel specific component of the apical structure of vertebrate motile cilia. Mol. Biol. Cell 19, 5338-5346, https://doi.org/10.1091/mbc.e08-07-0691

74 Watnick, T.J., Jin, Y., Matunis, E., Kernan, M.J. and Montell, C. (2003) A flagellar polycystin-2 homolog required for male fertility in Drosophila. Curr. Biol. 13, 2179-2184, https://doi.org/10.1016/j.cub.2003.12.002

75 Köttgen, M., Hofherr, A., Li, W., Chu, K., Cook, S., Montell, C. et al. (2011) Drosophila sperm swim backwards in the female reproductive tract and are activated via TRPP2 ion channels. PLOS ONE 6, e20031, https://doi.org/10.1371/journal.pone.0020031 
76 Lee, J., Moon, S., Cha, Y. and Chung, Y.D. (2010) Drosophila TRPN( = NOMPC) channel localizes to the distal end of mechanosensory cilia. PLoS ONE 5, e11012, https://doi.org/10.1371/journal.pone.0011012

77 Liu, Y.C., Couzens, A.L., Deshwar, A.R., McBroom-Cerajewski, L.D.B., Zhang, X., Puviindran, V. et al. (2014) The PPFIA1-PP2A protein complex promotes trafficking of Kif7 to the ciliary tip and Hedgehog signaling. Sci. Signal. 7, ra117, https://doi.org/10.1126/scisignal.2005608

78 Haycraft, C.J., Banizs, B., Aydin-Son, Y., Zhang, Q., Michaud, E.J. and Yoder, B.K. (2005) Gli2 and Gli3 localize to cilia and require the intraflagellar transport protein Polaris for processing and function. PLoS Genet. 1, e53, https://doi.org/10.1371/journal.pgen.0010053

79 Liu, W., Apagyi, K., McLeavy, L. and Ersfeld, K. (2010) Expression and cellular localisation of calpain-like proteins in Trypanosoma brucei. Mol. Biochem. Parasitol. 169, 20-26, https://doi.org/10.1016/j.molbiopara.2009.09.004

80 Akhmanova, A. and Steinmetz, M.0. (2015) Control of microtubule organization and dynamics: two ends in the limelight. Nat. Rev. Mol. Cell Biol. 16, 711-726, https://doi.org/10.1038/nrm4084

81 Taschner, M. and Lorentzen, E. (2016) The intraflagellar transport machinery. Cold Spring Harb. Perspect. Biol. 8, a028092, https://doi.org/10.1101/cshperspect.a028092

82 Tsao, C.-C. and Gorovsky, M.A. (2008) Different effects of Tetrahymena IFT172 domains on anterograde and retrograde intraflagellar transport. Mol. Biol. Cell 19, 1450-1461, https://doi.org/10.1091/mbc.e07-05-0403

83 Dean, S., Sunter, J.D. and Wheeler, R.J. (2017) TrypTag.org: a trypanosome genome-wide protein localisation resource. Trends Parasitol. 33, 80-82, https://doi.org/10.1016/j.pt.2016.10.009

84 Bangs, F. and Anderson, K.V. (2017) Primary cilia and mammalian Hedgehog signaling. Cold Spring Harb. Perspect. Biol. 9, https://doi.org/10.1101/cshperspect.a028175

85 He, M., Agbu, S. and Anderson, K.V. (2017) Microtubule motors drive hedgehog signaling in primary cilia. Trends Cell Biol. 27, 110-125, https://doi.org/10.1016/j.tcb.2016.09.010

86 Yue, Y., Blasius, T.L., Zhang, S., Jariwala, S., Walker, B., Grant, B.J. et al. (2018) Altered chemomechanical coupling causes impaired motility of the kinesin-4 motors KIF27 and KIF7. J. Cell Biol. 217, 1319-1334, https://doi.org/10.1083/jcb.201708179

87 Sengupta, P. (2017) Cilia and sensory signaling: the journey from "animalcules" to human disease. PLoS Biol. 15, e2002240, https://doi.org/10.1371/journal.pbio.2002240

88 Shah, A.S., Ben-Shahar, Y., Moninger, T.O., Kline, J.N. and Welsh, M.J. (2009) Motile cilia of human airway epithelia are chemosensory. Science $\mathbf{3 2 5}$ 1131-1134, https://doi.org/10.1126/science.1173869

89 Johnson, N.T., Villalón, M., Royce, F.H., Hard, R. and Verdugo, P. (1991) Autoregulation of beat frequency in respiratory ciliated cells: demonstration by viscous loading. Am. Rev. Respir. Dis. 144, 1091-1094, https://doi.org/10.1164/ajrccm/144.5.1091

90 Lorenzo, I.M., Liedtke, W., Sanderson, M.J. and Valverde, M.A. (2008) TRPV4 channel participates in receptor-operated calcium entry and ciliary beat frequency regulation in mouse airway epithelial cells. Proc. Natl. Acad. Sci. U.S.A. 105, 12611-12616, https://doi.org/10.1073/pnas.0803970105

91 O'Callaghan, C.L., Sikand, K., Rutman, A. and Hirst, R.A. (2008) The effect of viscous loading on brain ependymal cilia. Neurosci. Lett. 439, 56-60, https://doi.org/10.1016/..neulet.2008.04.095

92 Sanderson, M.J. and Dirksen, E.R. (1986) Mechanosensitivity of cultured ciliated cells from the mammalian respiratory tract: implications for the regulation of mucociliary transport. Proc. Natl. Acad. Sci. U.S.A. 83, 7302-7306, https://doi.org/10.1073/pnas.83.19.7302

93 Ogura, A. and Takahashi, K. (1976) Artificial deciliation causes loss of calcium-dependent responses in Paramecium. Nature 264, 170-172, https://doi.org/10.1038/264170a0

94 Solter, K.M. and Gibor, A. (1977) Evidence for role of flagella as sensory transducers in mating of Chlamydomonas reinhardi. Nature 265, 444-445, https://doi.org/10.1038/265444a0

95 Bloodgood, R.A. (2010) Sensory reception is an attribute of both primary cilia and motile cilia. J. Cell Sci. 123, 505-509, https://doi.org/10.1242/jcs.066308

96 Gluenz, E., Ginger, M.L. and McKean, P.G. (2010) Flagellum assembly and function during the Leishmania life cycle. Curr. Opin. Microbiol. 13 473-479, https://doi.org/10.1016/j.mib.2010.05.008

97 Goodenough, U.W. (1985) Structure of the Chlamydomonas agglutinin and related flagellar surface proteins in vitro and in situ. J. Cell Biol 101, 924-941, https://doi.org/10.1083/jcb.101.3.924

98 Mesland, D.A. (1980) Flagellar tip activation stimulated by membrane adhesions in Chlamydomonas gametes. J. Cell Biol. 84, 599-617, https://doi.org/10.1083/jcb.84.3.599

99 Demetsmets, R., Tomson, A.M., Stegwee, D. and Ende van den, H. (1990) Cell-cell coordination in conjugating Chlamydomonas gametes. Protoplasma 155, 188-199, https://doi.org/10.1007/BF01322628

100 Goodenough, U.W. and Jurivich, D. (1978) Tipping and mating-structure activation induced in Chlamydomonas gametes by flagellar membrane antisera. J. Cell Biol. 79, 680-693, https://doi.org/10.1083/jcb.79.3.680

101 Homan, W., Sigon, C., van den Briel, W., Wagter, R., de Nobel, H., Mesland, D. et al. (1987) Transport of membrane receptors and the mechanics of sexual cell fusion in Chlamydomonas eugametos. FEBS Lett. 215, 323-326, https://doi.org/10.1016/0014-5793(87)80170-9

102 Pasquale, S.M. (1987) Cyclic AMP functions as a primary sexual signal in gametes of Chlamydomonas reinhardtii. J. Cell Biol. 105, 2279-2292, https://doi.org/10.1083/jcb.105.5.2279

103 Wang, Q. and Snell, W.J. (2003) Flagellar adhesion between mating type plus and mating type minus gametes activates a flagellar protein-tyrosine kinase during fertilization in Chlamydomonas. J. Biol. Chem. 278, 32936-32942, https://doi.org/10.1074/jbc.M303261200

104 Wood, C.R. and Rosenbaum, J.L. (2015) Ciliary ectosomes: transmissions from the cell's antenna. Trends Cell Biol. 25, 276-285, https://doi.org/10.1016/j.tcb.2014.12.008

105 Wang, J. and Barr, M.M. (2018) Cell-cell communication via ciliary extracellular vesicles: clues from model systems. Essays Biochem. 62, 205-213, https://doi.org/10.1042/EBC20170085 
106 Wood, C.R., Huang, K., Diener, D.R. and Rosenbaum, J.L. (2013) The cilium secretes bioactive ectosomes. Curr. Biol. 23, 906-911, https://doi.org/10.1016/j.cub.2013.04.019 